\title{
Heteronuclear NMR studies of the specificity of the post-translational modification of biotinyl domains by biotinyl protein ligase
}

\author{
Pedro A. Reche*, Mark J. Howard ${ }^{1}$, R. William Broadhurst, Richard N. Perham ${ }^{2}$ \\ Cambridge Centre for Molecular Recognition, Department of Biochemistry, University of Cambridge, 80 Tennis Court Road, \\ Cambridge CB2 $1 G A$, UK
}

Received 25 May 2000; revised 16 June 2000; accepted 16 June 2000

Edited by Thomas L. James

\begin{abstract}
The lipoyl domains of 2-oxo acid dehydrogenase multienzyme complexes and the biotinyl domains of biotindependent enzymes have homologous structures, but the target lysine residue in each domain is correctly selected for posttranslational modification by lipoyl protein ligase and biotinyl protein ligase, respectively. We have applied two-dimensional heteronuclear NMR spectroscopy to investigate the interaction between the apo form of the biotinyl domain of the biotin carboxyl carrier protein of acetyl-CoA carboxylase and the biotinyl protein ligase (BPL) from Escherichia coli. Heteronuclear multiple quantum coherence NMR spectra of the ${ }^{15} \mathrm{~N}$ labelled biotinyl domain were recorded in the presence and absence of the ligase and backbone amide ${ }^{1} \mathrm{H}$ and ${ }^{15} \mathrm{~N}$ chemical shifts were evaluated. Small, but significant, changes in chemical shift were found in two regions, including the tight $\beta$-turn that houses the lysine residue targetted for biotinylation, and the $\beta$ strand 2 and the loop that precedes it in the domain. When compared with the three-dimensional structure, sequence alignments of other biotinyl and lipoyl domains, and mutagenesis data, these results give a clear indication of how the biotinyl domain is both recognised by BPL and distinguished from the structurally related lipoyl domain to ensure correct posttranslational modification. (c) 2000 Federation of European Biochemical Societies. Published by Elsevier Science B.V. All rights reserved.
\end{abstract}

Key words: Biotin; Biotinyl protein ligase; Protein domain; Molecular recognition; NMR spectroscopy

\section{Introduction}

Biotin is the prosthetic group of biotin-dependent enzyme complexes, which catalyse key metabolic reactions. Biotin is attached by covalent amide linkage to the $N^{6}$-amino group of a specific lysine residue, and acts by shuttling $\mathrm{CO}_{2}$ between the different active sites in these enzyme complexes [1]. In

\footnotetext{
*Corresponding author. Present address: DNAX Research Institute, 901 California Ave., Palo Alto, CA 94304-1104, USA.

Fax: (1)-650-496 1200. E-mail: reche@dnax.org

${ }^{1}$ Present address: Medical Research Council, Centre for Protein Engineering, Department of Chemistry, Lensfield Road, Cambridge CB2 1EW, UK

2 Also corresponding author. Fax: (44)-1223-333667; E-mail: r.n.perham@bioc.cam.ac.uk
}

Abbreviations: BCCP, biotin carboxyl carrier protein; BPL, biotinyl protein ligase; GCSH, the $\mathrm{H}$ protein of the glycine decarboxylase system; HMQC, heteronuclear multiple quantum coherence; LPL, lipoyl protein ligase; NMR, nuclear magnetic resonance
Escherichia coli, the only biotinylated protein is the biotin carboxyl carrier protein subunit (BCCP) of the acetyl-CoA carboxylase. The biotinyl group is attached post-translationally by the action of BirA [2], a bifunctional protein which, in addition to being a biotinyl protein ligase (BPL), represses the transcription of the genes involved in the biosynthesis of biotin [3].

The biotinylation reaction mediated by BPL occurs in a two-step manner. Initially, the ligase catalyses the activation of biotin to biotinyl-5'-AMP at the expense of ATP; following which, the biotinyl group is transferred to a specific lysine residue in the relevant enzyme. E. coli BPL can biotinylate apo-proteins from various sources and, in general, the BPL from one organism is found to recognise and modify biotindependent enzymes from other sources [4]. This promiscuous activity is due to the ability of BPL to modify a specific protein module, the biotinyl domain, which is present in all biotin-dependent enzymes [5,6]. The biotinyl domain encompasses approximately 80 residues surrounding the biotinyl-lysine, and in E. coli acetyl-CoA carboxylase is located at the Cterminus of the BCCP subunit [7]. The structure of the biotinyl domain of E. coli BCCP, as determined by X-ray crystallography [8] and heteronuclear NMR spectroscopy $[9,10]$, is that of a flattened $\beta$-barrel, comprising two sets of four antiparallel $\beta$-strands, with the biotinyl-lysine residue located in an exposed $\beta$-turn. This structure closely resembles those of the lipoyl domains in the dihydrolipoyl acyltransferase (E2) components of 2-oxo acid dehydrogenase multienzyme complexes [11-16], and of the H-protein in the glycine decarboxylase system (GCSH) [17]. The lipoyl domains also contain a modified (lipoylated) lysine residue, similarly located at the tip of the tight $\beta$-turn.

The lipoyl-lysine and biotinyl-lysine residues serve as swinging arms, ferrying substrate between the three active sites in their relevant enzyme complexes [18]. Attachment of lipoic acid to lipoyl domains and GCSH occurs post-translationally, and is mediated by specific enzymes. In $E$. coli, two such enzymes, encoded by the $\operatorname{lpl} A$ and $\operatorname{lip} B$ genes, respectively, have been reported [19]. The lipoylation reaction catalysed by LPL, the lipoyl protein ligase product of the $\operatorname{lpl} A$ gene, is analogous to that of the biotinylation reaction mediated by the BPL, with lipoyl-5'-AMP as the intermediate donor of the lipoyl moiety [20]. In contrast, LipB uses the endogenous lipoyl-acyl carrier protein (lipoyl-ACP) from the fatty acid biosynthesis pathway as the donor of the lipoyl group [21].

Correct positioning of the target lysine in the tight $\beta$-turn is essential for biotinylation of the biotinyl domain [22]. Several residues important for the recognition of the E. coli biotinyl domain by $E$. coli BPL have been identified, most notably the 
conserved Met residues flanking the target lysine [22], and the Glu residue at position -3 with respect the target lysine [23]. Additional data are also available for the biotinylation by $E$. coli BPL of biotinyl domains from other sources, including Propionibacterium shermanii transcarboxylase [24,25] and human propionyl-CoA carboxylase [26]. However, the mechanism by which BPL discriminates between the biotinyl and lipoyl domains remains elusive. In this paper, we have applied NMR spectroscopy to identify residues in the biotinyl domain that are perturbed by contact with BPL. With the help of a multiple sequence alignment of biotinyl and lipoyl domains and an analysis of the structure of the biotinyl domain, these results indicate that three-dimensional features on the biotinyl domain assist the BPL in recognising and distinguishing its substrate from the homologous lipoyl domains in the same cell.

\section{Materials and methods}

\subsection{Materials}

${ }^{15} \mathrm{~N}\left[\mathrm{NH}_{4} \mathrm{Cl}\right]$ and Celtone ${ }^{\circledR}$ were purchased from Martek Biosciences. $\mathrm{D}_{2} \mathrm{O}$ and bovine serum albumin (BSA) were from Sigma. Dimethylsilyl propionate (DSS) was from Melford Laboratories. Six percent crosslinked beaded agarose containing ImmunoPure ${ }^{\circledR}$ Immobilized Avidin was purchased from Pierce. All other chemicals were of analytical grade or better. E. coli host strains BL21(DE3) and BL21(DE3)plysS were from Novagen. Plasmid pTbpl carrying the gene encoding the BPL of $E$. coli was constructed by P. Reche and R.N. Perham (unpublished work).

\subsection{Purification of $B P L$ and the ${ }^{15} \mathrm{~N}$-labelled apo-biotinyl domain}

BPL was purified from E. coli BL21(DE3)plysS cells containing the plasmid pTbpl as described elsewhere in [22], and then dialysed against $20 \mathrm{mM}$ potassium phosphate buffer, $\mathrm{pH} 6.8 .{ }^{15} \mathrm{~N}$-labelled apo-biotinyl domain was prepared from $E$. coli BL21(DE3) cells containing the plasmid pGwtBCCP, which encodes the 82-residue biotinyl domain from the C-terminal end of the BCCP subunit of E. coli acetyl-CoA carboxylase as a fusion protein with glutathione $S$-transferase [22]. ${ }^{15} \mathrm{~N}$-labelling of the biotinyl domain domain was achieved by growing the cells on K-MOPS minimal medium [27] with ${ }^{15} \mathrm{~N}\left[\mathrm{NH}_{4} \mathrm{Cl}\right]$ as the main source of nitrogen, supplemented with $0.5 \%{ }^{15} \mathrm{~N}$-labelled Celtone ${ }^{\circledR}$. Purification of the domain was carried out as described previously [22]. A further affinity chromatography step using an agarose column containing ImmunoPure ${ }^{\circledR}$ Immobilized Avidin was introduced to eliminate any biotinylated form of the protein, basically as described by the manufacturer ( $1 \mathrm{mg}$ avidin was used per $3 \mathrm{mg}$ of biotinyl domain). The unretarded effluent containing the ${ }^{15} \mathrm{~N}$ apo-biotinyl domain was collected, dialysed against $20 \mathrm{mM}$ potassium phosphate buffer, $\mathrm{pH}$ 6.8, and concentrated in centripep-3 concentrators (Amicon). The molecular mass of 9076.5 \pm 1.0 Da determined by electrospray mass spectroscopy indicated that the biotinyl domain was unbiotinylated and homogeneously labelled. The concentrations of pure protein solutions were estimated by amino acid analysis [28].

\subsection{NMR spectroscopy and assignment procedure}

Two-dimensional NMR spectroscopy was performed using samples containing $1 \mathrm{mM}{ }^{15} \mathrm{~N}$-labelled apo-biotinyl domain in $20 \mathrm{mM}$ potassium phosphate buffer, $\mathrm{pH} 6.8,10 \% \mathrm{D}_{2} \mathrm{O}$ and $20 \mu \mathrm{M}$ dimethylsilyl propionate (DSS). All of the spectra were recorded at $298 \mathrm{~K}$ on a
Bruker AM500 spectrometer. Quadrature detection in the indirect dimensions was achieved using States-TPPI. The $\mathrm{H}_{2} \mathrm{O}$ resonance was suppressed by presaturation in all except the HMQC experiments where jump-return pulses on the ${ }^{1} \mathrm{H}$ channel with a delay of $125 \mu \mathrm{s}$ were utilised. Sequence-specific resonance assignments [9] were confirmed by standard methods [29] using the experiments listed in Table 1. Data processing and spectral analysis were carried out on a Silicon Graphics Indy workstation using the programs AZARA (W. Boucher, unpublished work) and ANSIG 3.3 [30], respectively. Both programs are available by anonymous ftp to ftp.bio.cam.ac.uk in the directory $\sim$ ftp/pub.

\section{Results and discussion}

\subsection{The interaction between the biotinyl domain and BPL can be monitored by heteronuclear NMR spectroscopy}

It is clear that protein-protein interactions underlie the process by which BPL distinguishes the biotinyl domain from the homologous lipoyl domains and selects just a single lysine for biotinylation. In this paper, we describe studies of the interaction between the biotinyl domain and BPL by means of heteronuclear NMR spectroscopy, using well-established techniques for identifying protein-protein contacts [31,32]. We have compared the ${ }^{15} \mathrm{~N}-{ }^{1} \mathrm{H}$ heteronuclear multiple quantum correlation (HMQC) spectrum of the ${ }^{15} \mathrm{~N}$-labelled apo-biotinyl domain with that obtained for the same domain in the presence of BPL. First, the assignment of resonances in the HMQC spectrum of $1 \mathrm{mM}$ unbiotinylated ${ }^{15} \mathrm{~N}$-labelled biotinyl domain [9] was confirmed using spin systems and sequential assignments identified from z-filtered TOCSY, NOESY, HSQC-NOESY and HSQC-TOCSY experiments. On addition of BPL up to a final concentration $0.25 \mathrm{mM}$, small changes in backbone amide ${ }^{15} \mathrm{~N}$ and ${ }^{1} \mathrm{H}$ chemical shifts were observed for some crosspeaks in the HMQC spectrum of the apo-biotinyl domain (spectrum not shown). The chemical shift changes experienced by residues in the biotinyl domain are summarised in Fig. 1A,B. The largest chemical shift changes were $0.024 \mathrm{ppm}$ for ${ }^{1} \mathrm{H}$ (Asn-125) (Fig. 1A) and $0.13 \mathrm{ppm}$ for ${ }^{15} \mathrm{~N}$ (Met-123) (Fig. 1B). The most noticeable changes were observed for resonances from the backbone amides of residues Met-87, Gly-89, Thr-90, Met-121, Lys-122, Met-123 and Asn125 (the numbering is that of the E. coli BCCP). Smaller, but significant, changes were observed in residues Glu-119, Met124, Gln-126 and Glu-128. In favourable circumstances, these changes in chemical shift should result from protein-protein contacts and point to residues that are involved in the interaction with the ligase. However, given that small chemical shift changes may also be due to minute buffer variations or temperature changes, the experiments were repeated. Further HMQC spectra of the apo-biotinyl domain were run with lower concentrations of BPL, which showed intermediate changes in chemical shifts and line widths for the affected cross peaks (data not shown). These results demonstrate that although the observed chemical shift changes are small, they are significant and are caused by an interaction with the

Table 1

NMR spectra recorded for resonance assignment and studying interactions

\begin{tabular}{lclll}
\hline Experiment & $\tau_{\text {mix }}(\mathrm{ms})$ & Data size $($ complex points) & $\tau_{\max }(\mathrm{ms})$ & References \\
\hline 2D $\left[{ }^{15} \mathrm{~N},{ }^{15} \mathrm{H}\right]$-HMQC & & $64 \times 1024$ & $128(\mathrm{~N}), 127(\mathrm{H})$ & {$[35]$} \\
2D NOESY & 100 & $256 \times 2048$ & $32(\mathrm{H}), 256(\mathrm{H})$ & {$[36]$} \\
2D TOCSY & 59 & $256 \times 2048$ & $32(\mathrm{H}), 256(\mathrm{H})$ & {$[37]$} \\
2D [ $\left.{ }^{15} \mathrm{~N},{ }^{15} \mathrm{H}\right]$-HSQC-NOESY & 150 & $150 \times 1024$ & $300(\mathrm{~N}), 127(\mathrm{H})$ & {$[38]$} \\
2D $\left[{ }^{15} \mathrm{~N},{ }^{15} \mathrm{H}\right]$-HSQC-TOCSY & 59 & $150 \times 1024$ & $300(\mathrm{~N}), 127(\mathrm{H})$ & {$[38]$} \\
\hline
\end{tabular}



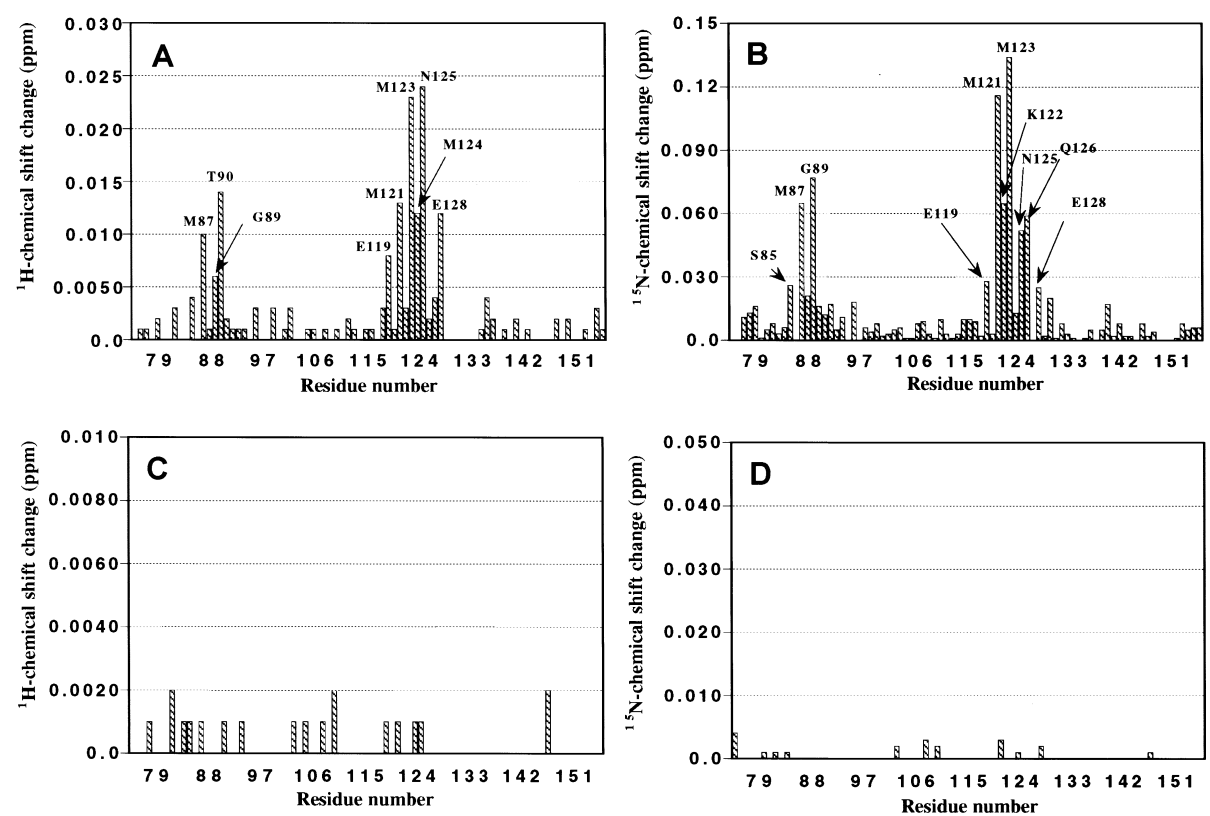

Fig. 1. Analysis of chemical shift changes experienced by resonances of the biotinyl domain. Chemical shift differences between the $2 \mathrm{D}{ }^{15} \mathrm{~N}-{ }^{1} \mathrm{H}$ HMQC spectra of apo-biotinyl domain recorded in the absence (A) and presence of BPL (B) and in the absence (C) and presence of BSA (D). ${ }^{1} \mathrm{H}$ and ${ }^{15} \mathrm{~N}$ backbone chemical shift changes are plotted against residue number (BCCP numbering). Residues with resonances that undergo significant changes are indicated.

ligase that is in fast exchange on the chemical shift time scale. In addition, as a control, an HMQC spectrum of the apobiotinyl domain was acquired in the presence of $0.45 \mathrm{mM}$ BSA. The resulting changes in chemical shift from the values observed for the apo-biotinyl domain alone were smaller than 0.003 and $0.005 \mathrm{ppm}$ for the ${ }^{1} \mathrm{H}$ and ${ }^{15} \mathrm{~N}$ dimensions, respectively (Fig. 1C,D). This indicates that there is no non-specific binding of the apo-biotinyl domain to BSA. More importantly, it implies that the changes observed in the HMQC spectrum in the presence of BPL are significant and the result of a specific interaction.

\subsection{Two regions in the primary structure of the biotinyl domain interact with BPL}

Inspection of changes in both ${ }^{1} \mathrm{H}$ and ${ }^{15} \mathrm{~N}$ chemical shifts in the presence of BPL reveals a similar pattern. The residues with the largest chemical shift changes cluster in two regions of the primary structure of the biotinyl domain (Fig. 1). The first cluster comprises strand $\beta 2$ and the loop that precedes it, particularly residues Met-87, Gly-89 and Thr-90. Pro-86 may also belong to this cluster, but is not detected in the HMQC spectrum because proline residues lack an amide proton. Less significant changes were seen for Val-88, which is also found in this section of the primary sequence (Fig. 1). The second cluster contains the $\beta$-hairpin turn that displays the target lysine residue (Lys-122), with residues Glu-119, Met-121, Met-123, Met-124, Asn-125, Gln-126 and Glu-128 showing the greatest changes in their nuclear environments. Ala-120 is also in this region, but was excluded from the analysis because of overlap with the resonance from Ser-142. Although the two clusters are remote in the primary sequence of the biotinyl domain, the tertiary fold brings them into close proximity (Fig. 2), consistent with correct folding of the domain being essential for biotinylation. Single site mutations in the hydrophobic core of the biotinyl domain can hinder biotiny- lation of the surface lysine residue by $E$. coli BPL, presumably because the protein unfolds [23].

\subsection{Identifying relevant residues in the interaction of the biotinyl domain with $B P L$}

The current study has identified the site on the surface of the biotinyl domain that interacts with E. coli BPL (Fig. 2). Because E. coli BPL can biotinylate the biotinyl domains from diverse sources [4], a combination of the NMR results with an amino acid sequence comparison of various biotinyl domains should further highlight key residues for the interaction with BPL. Fig. 3 reveals that residues Met-87, Gly-89, Glu-119, Met-121, Lys-122 and Met-123, should have a role in the interaction, since they all experience significant changes in chemical shift in the presence of BPL and are conserved among biotin-dependent enzymes.

The role of the target lysine (Lys-122) is obviously crucial, and mutagenesis studies provide an indication of the importance of the other residues. Thus, substitution of Glu-119 for lysine renders the $E$. coli biotinyl domain virtually inactive as a substrate for BPL [23], and specific mutations of either of the Met residues flanking Lys-122 severely reduce the ability of the domain to accept biotinylation [22]. In addition, a mutation of the site equivalent to Met-87 in the biotinyl domain of the $\alpha$-subunit from human propionyl-CoA carboxylase (Met641Lys) dramatically reduces the rate of biotinylation by $E$. coli BPL [26]. There are currently no mutagenesis data available for Gly-89. Chapman-Smith et al. [23] have reported that mutation of Glu-147 to lysine causes a considerable reduction in the rate of biotinylation of the $E$. coli biotinyl domain. We do not see major chemical shift changes for the backbone amide resonance of this residue, but it is located adjacent to the biotinylation interface that we have identified on the biotinyl domain (Fig. 2).

Residues that display significant chemical shift changes, but 
A

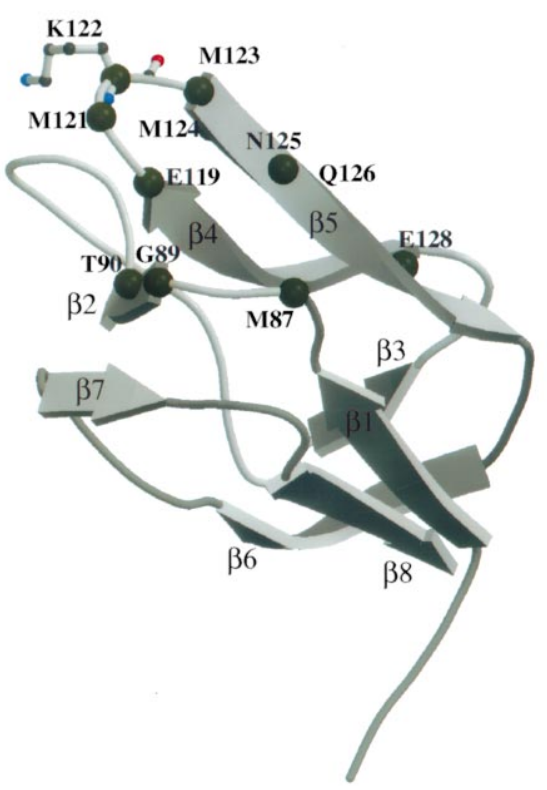

B

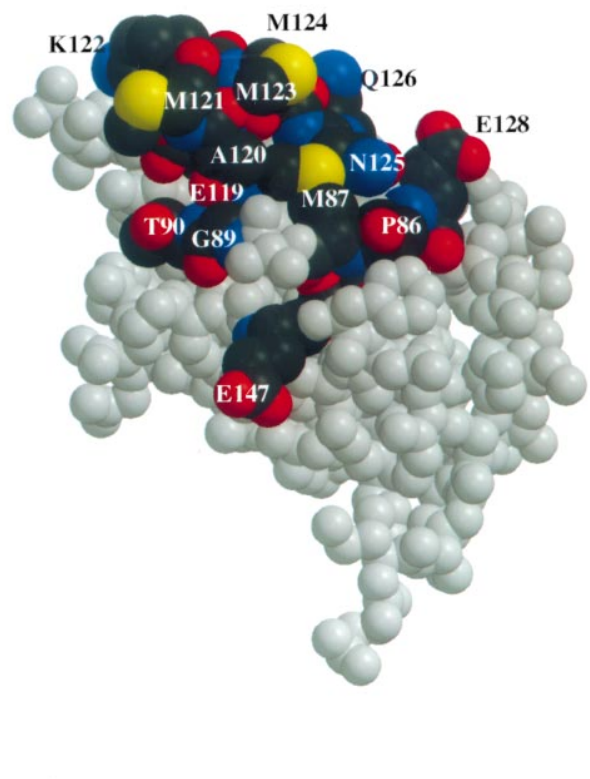

Fig. 2. Mapping the residues in the biotinyl domain that make contact with BPL. Residues that exhibited significant changes in chemical shift in the presence of BPL are highlighted in a backbone schematic (A) and a space filling representation (B) of the biotinyl domain structure. Glu-147 is also identified in B. We did not detect chemical shift changes for Glu-147, but we have included this residue here because of mutagenesis data [23] referred to in the text. Pro-14 and Ala-48 residues are also included in B for reasons explained in the text. This figure was made using Molscript [39], followed by image rendering with Raster3D [40]. Coordinates are taken from Athappilly and Hendrickson [8].

are not conserved, probably do not perform crucial roles in the interaction with the ligase. The rate of biotinylation by BPL is not affected in a biotinyl domain in which residues Met-124, Asn-125, Gln-126 and Glu-128 have been changed to Ser, Met, Glu, and Pro, respectively [6]. It follows that the contribution made by the side chains of these residues to the interaction interface cannot be unique. Alternatively, it may be that the chemical shift changes observed for Met-124, Asn125 , Gln-126 and Glu-128 are due to a relaxation in the structure of the biotinyl domain, rather than a direct result of being in contact with BPL.

\subsection{Evidence for topographic recognition of the biotinyl domain by $B P L$}

Of all the residues in the biotinyl domain that we have identified as playing a role in the interaction with BPL, only the methionines flanking the target lysine (in the MKM motif) are well exposed to the solvent (Fig. 2). These highly conserved residues may act as a molecular signal for biotinylation, but, surprisingly, it has been reported that they can be replaced by Leu without detriment to the biotinylation rates of the biotinyl domains from both $P$. shermanii transcarboxylase [25] and human propionyl-CoA carboxylase [26]. However, the MKM motif of the biotinyl domain cannot by itself specify biotinylation, as it has been shown that replacing the DKA sequence found at the tip of the corresponding hairpin loop in the lipoyl domain from Bacillus sterarothermophillus pyruvate dehydrogenase complex with MKM does not make the lipoyl domain a substrate for BPL in vivo [33]. Thus, it is apparent that recognition of the biotinyl domain is a rather complex process, relying on more than just a region of its primary sequence or isolated molecular signals.
The three-dimensional structure and molecular surface of the biotinyl domain (Fig. 2) indicate that the residues we have identified as relevant for the interaction with BPL are also important for the tertiary structure of the biotinyl domain. Met- 87 is partially buried and contributes to the hydrophobic core, while Gly-89 contributes to several hydrogen bonds and marks the beginning of the $\beta 2$-strand [8] (Fig. 2). In addition, the side chain of the conserved Pro-86 (Fig. 2) is not prominent on the molecular surface of the biotinyl domain, but partially buried. Mutagenesis data indicate that Pro-86 is important for biotinylation of the human propionyl-CoA carboxylase biotinyl domain [26]. The conserved residue Glu-119 does not play a key role in the structure of the biotinyl domain; neither is it displayed prominently on the molecular surface of the domain. Its contribution to the interaction with BPL seems to be electrostatic in nature, as already pointed out elsewhere [23].

Overall, these observations indicate that BPL is interacting with a region of the biotinyl domain whose three-dimensional shape (topography) is largely dictated by the tertiary fold. Recognition of the fold of the biotinyl domain by BPL explains the observed promiscuity of the ligase and liberates it from the need for a single molecular signal for biotinylation. These findings provide a striking contrast with other forms of post-translational modification, such as phosphorylation, where the primary structure surrounding the target residue can be of crucial importance.

\subsection{Three-dimensional features enable BPL to distinguish between biotinyl and lipoyl domains}

Lipoyl domains are structurally homologous to biotinyl domains, with their target lysine residues positioned in comparable $\beta$-turns. Correct positioning of the target lysine in 

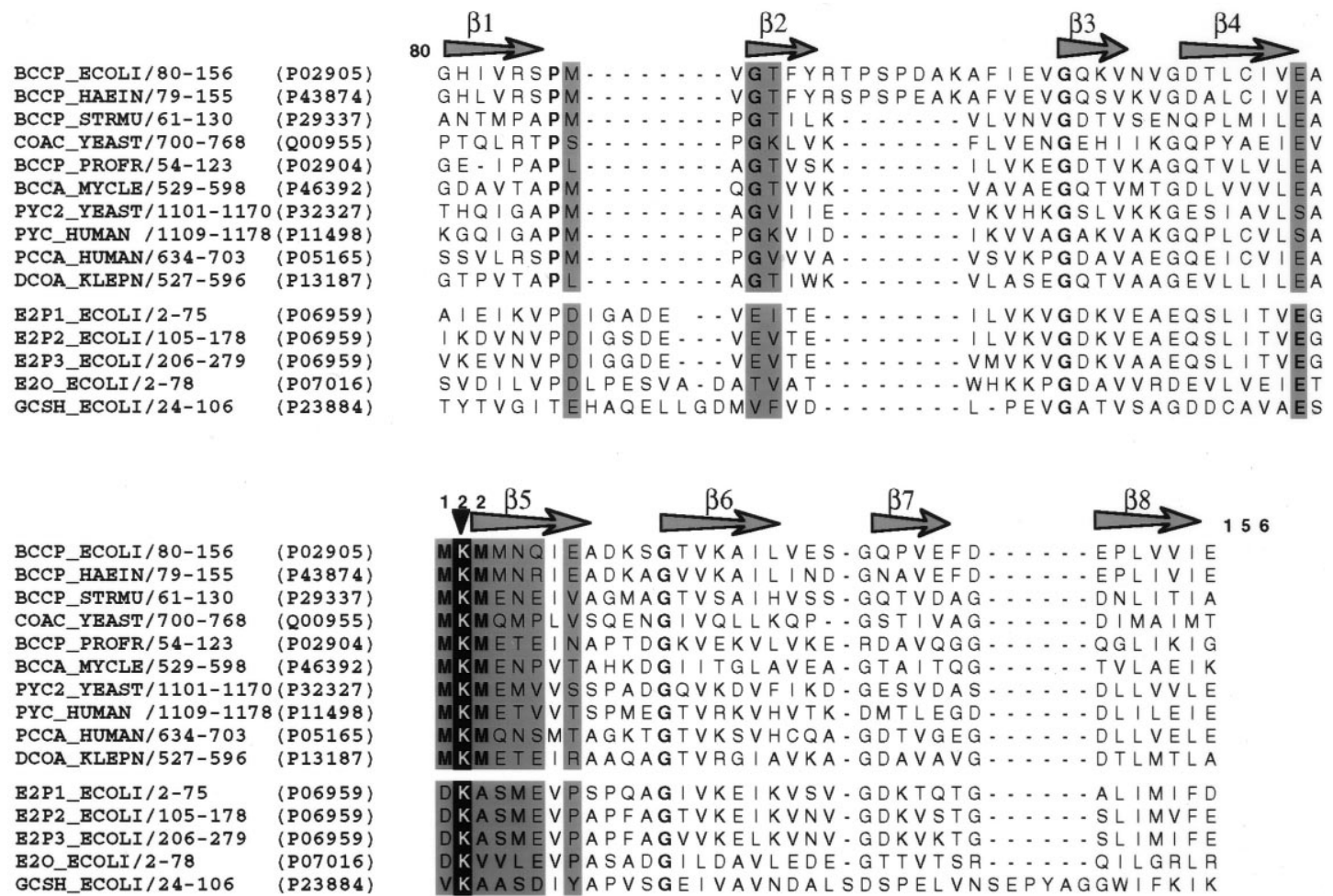

Fig. 3. Structure-based sequence alignment of various biotinyl domains and proteins that are found lipoylated in $E$. coli. Aligned sequences are noted by their SwissProt identifiers (protein name_specie) and are grouped into biotinylated (top) and lipoylated (bottom) proteins. For each sequence, the SwissProt accession number is given, and the extent of the aligned region is indicated. The following classes of biotinylated proteins are aligned: BCCP, biotin carboxyl carrier protein; BCCA, acetyl/propionyl-CoA carboxylase; COAC, acetyl-CoA carboxylase; PYC and PYC2, pyruvate carboxylase: PCCA, $\alpha$ chain of propionyl-CoA carboxylase; DCOA, $\alpha$ chain of oxaloacetate decarboxylase. Lipoylated proteins aligned are the following: E2P1_ECOLI, E2P2_ECOLI, and E2P3_ECOLI; outer, middle and inner, respectively, lipoyl domains of the E2 component of the E. coli pyruvate dehydrogenase complex; E2O_ECOLI, lipoyl domain of the E2 component of the E. coli 2-oxoglutarate dehydrogenase complex; and GCSH_ECOLI, the lipoylated H-protein component of the $E$. coli glycine decarboxylase system. The $\beta$-strands of the biotinyl domain of E. coli BCCP are represented by arrows above the amino acid sequence. Identical residues between biotin-containing enzymes and lipoylated proteins are shown in bold. The target lysine for modification is shaded in black. The residue number (122) of the target lysine in the E. coli biotinyl domain is shown on top of its sequence (BCCP numbering). Positions occupied by residues of the E. coli biotinyl domain with the most significant changes in chemical shift in the presence of BPL are shaded in grey. The seed of the structural alignment was obtained from the FSSP database (http://www2.ebi.ac.uk/dali/fssp/).

this tight $\beta$-turn is essential in targeting both the lipoylation of lipoyl domains [33] and the biotinylation of biotinyl domains [22]. Therefore, there must be key differences between the biotinyl and lipoyl domains that allow the relevant protein ligases to distinguish between them for the purposes of posttranslational modification. Sequence differences between the biotinyl and lipoyl domains in terms of the residues identified as relevant to biotinylation should assist BPL in recognising its correct substrate. Thus, BPL will not biotinylate a biotinyl domain with DKA replacing the MKM motif in the tight $\beta$ turn [22]. This change alone is enough to prevent biotinylation of the three lipoyl domains from the $E$. coli pyruvate dehydrogenase complex, given that they all contain the DKA motif (Fig. 3). In the GCSH protein and the lipoyl domain from the 2-oxoglutarate dehydrogenase complex, the equivalent sequence motifs at the $\beta$-turn are VKA and DKV, respectively (Fig. 3), but whether those motifs are also sufficient to prevent biotinylation remains to be proved. The structural basis of the ability of BPL to discriminate in favour of the biotinyl domain can be inferred from the fold-driven nature of the recognition that we have outlined.

The NMR experiments described here indicate that the $\beta 2$ strand and the preceding loop contribute to the interaction surface of the biotinyl domain with BPL (Fig. 2). Sequence alignments indicate that the lipoyl domains and the GCSH possess a sequence insertion in this region (Fig. 3). In the GCSH, an $\alpha$-helix is observed [17], and in the lipoyl domains of the 2-oxoglutarate and pyruvate dehydrogenase complexes this section is occupied by a loop of variable length (Fig. 3). These features would dramatically modify the surface topography of the lipoyl domains and the H-protein at this potential biotinylation interface, ensuring that they are not recognised by BPL.

Structural cues also seem to dominate the recognition of lipoyl domains by their lipoylating ligases. In the $E$. coli proteins, there is a sequence insertion between strands $\beta 2$ and $\beta 3$ of the biotinyl domain that forms a protruding 'thumb' [8-10] that is not present in the lipoyl domain (Fig. 3). This region provides protection from aberrant lipoylation of the biotinyl domain, since deletion of the thumb makes the biotinyl domain a substrate for lipoylation, both in vivo and in vitro [6]. It is interesting to note that in the current study no significant chemical shifts were observed for residues located in the protruding thumb of the biotinyl domain. Together with the finding that deletion of the thumb did not have a major affect on the pattern of biotinylation [6], this suggests that the prospective biotinylation and lipoylation interfaces of the domain are different. 


\section{Conclusions}

Heteronuclear NMR has been successfully used to monitor the interaction between the biotinyl domain and BPL of $E$. coli. This has pinpointed the molecular signal that enables the biotinyl domain to be biotinylated, giving particular importance to the role of the methionine residues that flank the target lysine residue. Inspection of these and other data indicates that the surface topography of the region near the biotinylation site, which is dictated by the tertiary fold, plays a key role in the recognition of the domain by BPL. The structure of $E$. coli BPL has been solved by X-ray crystallography [34]. The present work should provide useful information for future docking studies of the biotinyl domain and its ligase.

Acknowledgements: We thank the Federation of European Biochemical Societies for a Fellowship awarded to P.R. and the BBSRC for a research grant awarded to R.N.P. The core facilities of the Cambridge Centre for Molecular Recognition are supported by the BBSRC and The Wellcome Trust.

\section{References}

[1] Knowles, J.R. (1989) Annu. Rev. Biochem. 58, 195-221.

[2] Barker, D.F. and Campbell, A.M. (1981) J. Mol. Biol. 146, 451467.

[3] Cronan Jr., J.E. (1989) Cell 58, 427-428.

[4] Cronan Jr., J.E. (1990) J. Biol. Chem. 265, 10327-10333.

[5] Samols, D., Thornton, C.G., Murtif, V.L., Kumar, G.K., Haase, F.C. and Wood, H.G. (1988) J. Biol. Chem. 263, 6461-6464.

[6] Reche, P. and Perham, R.N. (1999) EMBO J. 18, 2673-2682.

[7] Li, S.-J. and Cronan Jr., J.E. (1991) J. Biol. Chem. 267, 855-863.

[8] Athappilly, F.K. and Hendrickson, W.A. (1995) Structure 3, $1407-1419$.

[9] Roberts, E.L., Shu, N., Howard, M.J., Broadhurst, R.W., Chapman-Smith, A., Wallace, J.C., Morris, T., Cronan Jr., J.E. and Perham, R.N. (1999) Biochemistry 38, 5045-5053.

[10] Yao, X., Soden Jr., C., Summers, M.F. and Beckett, D. (1999) Protein Sci. 8, 307-317.

[11] Dardel, F., Davis, A.L., Laue, E.D. and Perham, R.N. (1993) J. Mol. Biol. 229, 1037-1048.

[12] Green, J.D.F., Laue, E.D., Perham, R.N., Ali, S.T. and Guest, J.R. (1995) J. Mol. Biol. 248, 328-343.

[13] Ricaud, P.M., Howard, M.J., Roberts, E.L., Broadhurst, R.W. and Perham, R.N. (1996) J. Mol. Biol. 264, 179-190.

[14] Berg, A., Vervoort, J. and de Kok, A. (1996) J. Mol. Biol. 261, 432-442.

[15] Berg, A., Vervoort, J. and de Kok, A. (1997) Eur. J. Biochem. $244,352-360$.
[16] Howard, M.J., Fuller, C., Broadhurst, R.W., Quinn, J., Diamond, A.G., Tang, J., Perham, R.N. and Yeaman, S. (1998) Gastroenterology 115, 139-146.

[17] Pares, S., Cohen-Addad, C., Sieker, L., Neuburger, M. and Douce, R. (1994) Proc. Natl. Acad. Sci. USA 91, 4850-4853.

[18] Perham, R.N. and Reche, P.A. (1998) Biochem. Soc. Trans. 26, 299-303.

[19] Morris, T.W., Reed, K.E. and Cronan Jr., J.E. (1995) J. Bacteriol. $177,1-10$.

[20] Morris, T.W., Reed, K.E. and Cronan Jr., J.E. (1994) J. Biol. Chem. 269, 16091-16100.

[21] Jordan, S.W. and Cronan Jr., J.E. (1997) J. Biol. Chem. 272, 17903-17906.

[22] Reche, P., Li, Y.-L., Fuller, C., Eichhorn, K. and Perham, R.N. (1998) Biochem. J. 329, 587-596.

[23] Chapman-Smith, A., Morris, T.W., Wallace, J.C. and Cronan Jr., J.E. (1999) J. Mol. Biol. 274, 1449-1457.

[24] Murtif, V.L. and Samols, D. (1987) J. Biol. Chem. 262, $11813-$ 11816.

[25] Shenoy, B.C., Paranjape, S., Murtif, V.L., Kumar, G.K., Samols, D. and Wood, H.G. (1988) FASEB J. 2, 2505-2511.

[26] Leon-Del-Rio, A. and Gravel, R.A. (1994) J. Biol. Chem. 269, 22964-22968.

[27] Niedhardt, F.C., Bloch, P.L. and Smith, D.F. (1974) J. Bacteriol. 119, 736-747.

[28] Packman, L.C., Borges, A. and Perham, R.N. (1988) Biochem. J. $252,79-86$.

[29] Clore, G.M. and Gronenborn, A.M. (1989) Crit. Rev. Biochem. Mol. Biol. 24, 479-564.

[30] Kraulis, P.J. (1989) J. Magn. Res. 84, 627-633.

[31] Cheng, Y., Reizer, J., Saier, M.H., Fairbrother, W.J. and Wright, P.E. (1993) Biochemistry 32, 32-37.

[32] Gronenborn, A.M. and Clore, G.M. (1993) J. Mol. Biol. 233, 331-335.

[33] Wallis, N.G. and Perham, R.N. (1994) J. Mol. Biol. 236, 209216.

[34] Wilson, K.P., Shewchuk, L.M., Brennan, R.G., Otsuka, A.J. and Matthews, B.W. (1992) Proc. Natl. Acad. Sci. USA 89, 92579261.

[35] Bax, A., Ikura, M., Kay, L.E., Torchia, D.A. and Tschudin, R. (1990) J. Magn. Reson. 86, 304-318.

[36] Jeener, J., Meier, B.H., Bachmann, P. and Ernst, R.R. (1979) J. Chem. Phys. 71, 4546-4553.

[37] Rance, M.J. (1987) Magn. Reson. 74, 557-564.

[38] Norwood, T.J., Boyd, J., Heritage, J.E., Shoffe, N. and Campbell, I.D. (1990) J. Magn. Reson. 87, 488-501.

[39] Kraulis, P.J., Domaille, P.J., Cambell-Burk, S.L., van Aken, T. and Laue, E.D. (1994) Biochemistry 33, 3515-3531.

[40] Merritt, E.A. and Murphy, M.E.P. (1994) Acta Cryst. D50, 869873. 